\title{
Collisional excitation of sulfur dioxide in cold molecular clouds ${ }^{\star}$
}

\author{
J. Cernicharo ${ }^{1}$, A. Spielfiedel ${ }^{2}$, C. Balança ${ }^{2}$, F. Dayou $^{1}$, M.-L. Senent ${ }^{1}$, N. Feautrier ${ }^{2}$, \\ A. Faure ${ }^{3}$, L. Cressiot-Vincent ${ }^{1,2}$, L. Wiesenfeld ${ }^{3}$, and J. R. Pardo ${ }^{1}$ \\ ${ }^{1}$ CAB. INTA-CSIC. Department of Astrophysics. Crta Torrejón km 4. Torrejón de Ardoz, Madrid, Spain \\ e-mail: jcernicharo@cab.inta-csic.es \\ 2 LERMA and UMR 8112 of CNRS, Observatoire de Paris-Meudon, 92195 Meudon Cedex, France \\ e-mail: annie.spielfiedel@obspm.fr \\ 3 Laboratoire d'Astrophysique de Grenoble, UMR 5571-CNRS, Université Joseph Fourier, Grenoble, France \\ e-mail: alexandre. faure@obs.ujf-grenoble.fr
}

Received 26 November 2010 / Accepted 5 April 2011

\begin{abstract}
We present collisional rate coefficients for $\mathrm{SO}_{2}$ with ortho and para molecular hydrogen for the physical conditions prevailing in dark molecular clouds. Rate coefficients for the first 31 rotational levels of this species (energies up to $55 \mathrm{~K}$ ) and for temperatures between 5 and $30 \mathrm{~K}$ are provided. We have found that these rate coefficients are about ten times more than those previously computed for $\mathrm{SO}_{2}$ with helium. We calculated the expected emission of the centimeter wavelength lines of $\mathrm{SO}_{2}$. We find that the transition connecting the metastable $2_{02}$ level with the $1_{11}$ one is in absorption against the cosmic background for a wide range of densities. The $4_{04}-3_{13}$ line is found to be inverted for densities below a few $10^{4} \mathrm{~cm}^{-3}$. We observed the $1_{11}-2_{02}$ transition with the $100 \mathrm{~m}$ Green Bank Telescope towards some dark clouds. The line is observed, as expected, in absorption and provides an abundance of $\mathrm{SO}_{2}$ in these objects of a few $10^{-10}$. The potential use of millimeter lines of $\mathrm{SO}_{2}$ as tracers of the physical conditions of dark clouds is discussed.
\end{abstract}

Key words. molecular processes - ISM: molecules - ISM: abundances

\section{Introduction}

The observation of molecular emission at millimeter and infrared wavelengths, supplemented by careful and detailed modeling, is a powerful tool for investigating the physical and chemical conditions of astrophysical objects. Since the detection of sulfur dioxide, $\mathrm{SO}_{2}$, by Snyder et al. (1975), this molecule has been found to be very abundant (relative to $\mathrm{H}_{2}$ ) in warm molecular clouds (Schilke et al. 2001; Schloerb et al. 1983). It has also been observed towards cold dark clouds with an abundance of a few $10^{-9}$ (Irvine et al. 1983; Dickens et al. 2000). Interpretation of the data requires a precise knowledge of the collisional rates of the molecule under study and the main colliders in the cloud, i.e., molecular hydrogen and helium. Green (1995) has computed these rate coefficients ${ }^{1}$ for the system $\mathrm{SO}_{2}-\mathrm{He}$ for temperatures between $25-125 \mathrm{~K}$ for the first 50 rotational levels of this molecule (i.e., for levels with energies below $90 \mathrm{~K}$ ). These rates are useful for clouds with temperatures around 25-40 K. However, for warm molecular clouds in which lines involve energy levels of several hundred $\mathrm{K}$, or in O-rich evolved stars that show lines with levels of more than one thousand Kelvin, adequate radiative transfer models cannot be carried out owing to the lack of collisional rates at these high temperatures. Moreover, for dark clouds it will be extremely difficult to extrapolate the rates from $25 \mathrm{~K}$ to $10 \mathrm{~K}$, which is the typical

* Tables 5 and 6 are only available at the CDS via anonymous ftp to cdsarc.u-strasbg.fr $(130.79 .128 .5)$ or via

http://cdsarc.u-strasbg.fr/viz-bin/qcat?J/A+A/531/A103

1 Please note that, to simplify the writing, "rates" refers to "rate coefficients" throughout the paper. temperature of these objects, as many resonances occur at low energies and may make a strong contribution to collisional rates when the temperature decreases.

$\mathrm{SO}_{2}$ is one of the molecular species that is being observed with the Herschel Space Observatory in the submillimeter and far-infrared domains thereby increasing the number of transitions and also increasing the energies of observed rotational levels. The new generation of receivers for ground-based telescopes will permit sensitive line surveys to be carried out in dark clouds in the centimeter and millimeter domains. It would be a pity if all these observational efforts do not provide outstanding science because of the lack of collisional rates. In this paper, we provide collisional rates for temperatures from 5 to $30 \mathrm{~K}$ for the first 31 levels of $\mathrm{SO}_{2}$. In a subsequent paper, we will provide calculations for high temperatures and for a large number of rotational levels.

Considering the sensitivity of the radiative transfer models to the collisional rate coefficients, it is very important to provide accurate values for these coefficients. Thanks to recent progress in quantum chemistry calculations of the potential energy interaction between the two collision partners, scattering calculations based on the quantum close coupling (CC) method can provide the best accuracy to date, a few percent (Lique et al. 2010).

The methods and the results for the collisional rates are described in Sect. 2. In Sect. 3 we discuss the scope of the new collisional rates and predict the expected emission of several lines of $\mathrm{SO}_{2}$ in dark clouds. In particular, we show that the line $1_{11}-2_{02}$ could be in absorption against the cosmic background and that the $4_{04}-3_{13}$ line could be a strong maser in dark clouds. 


\section{Inelastic cross sections and rates for excitation of $\mathrm{SO}_{2}$ by para and ortho $\mathrm{H}_{2}$}

\subsection{Potential energy surface and general procedure}

All calculations presented below were performed with rigid molecules using the expansion of the 5D-PES described in Spielfiedel et al. (2009) where full details can be found. We used the conventions of Phillips et al. (1996) in defining the $\mathrm{SO}_{2}-\mathrm{H}_{2}$ intermolecular potential in the body-fixed frame. The $\mathrm{SO}_{2}$ molecule lies in the $x z$ plane with the symmetry axis along $z$. The rigid body PES involves five coordinates: the collision coordinate, i.e. the vector from $\mathrm{SO}_{2}$ center of mass to $\mathrm{H}_{2}$ center of mass, described by the spherical polar coordinates $(R, \theta, \phi)$, and the $\left(\theta^{\prime}, \phi^{\prime}\right)$ angles giving the $\mathrm{H}_{2}$ orientation relative to the $\mathrm{SO}_{2}$ molecule in the body-fixed system.

Following the strategy developed for the $\mathrm{H}_{2} \mathrm{O}-\mathrm{H}_{2}$ system (Valiron et al. 2008), the PES was calculated at the $\operatorname{CCSD}(\mathrm{T})$ level using a three-step procedure with basis sets of increasing accuracy and a complete basis set (CBS) extrapolation. Extensive calculations of a reference PES were performed for 49 intermolecular distances and 8074 sets of angles for each distance using the $a V D Z$ basis of Woon et al. (1994) and bond functions from William et al. (1995). Corrections, including CBS extrapolation, were performed to obtain the final intermolecular potential. The global minimum was found to be $V_{\min }=-192.723758 \mathrm{~cm}^{-1}$ for $R=6.147979$ Bohr, $\theta=91.721778^{\circ}, \phi=90.0^{\circ}, \theta^{\prime}=90.0^{\circ}$ and $\phi^{\prime}=0^{\circ}$. Seven secondary minima were also found. The angular expansion of the PES was obtained from the final very accurate set as a least square fit of the ab initio values for each of 49 intermolecular distances, leading to 129 angular functions that are specifically adapted to quantum calculations. The selection was found to reproduce the ab initio values within $1 \mathrm{~cm}^{-1}$ for distances $R$ greater than $6.5 a_{0}$. This result indicates the high quality of the angular fit in the long-range and van der Waals minimum regions of the interaction.

Close coupling calculations were performed with the mixed MPI and OpenMP ${ }^{2}$ extension of the MOLSCAT V14 package (Hutson \& Green 1994). The asymmetric top wave functions of ${ }^{32} \mathrm{SO}_{2}$ were obtained from the rotational constants and distortion constants given by Lovas (1978) and evaluated in the same coordinate system as was used to describe the PES. The rotational constants about the $x, y$, and $z$ axes are 2.027354, 0.29353 , and $0.344170 \mathrm{~cm}^{-1}$. The adopted centrifugal distortion constants (for a non-rigid asymmetric rotor) for $\mathrm{SO}_{2}$ in its vibrational ground state are $-7.2235 \times 10^{-7}, 3.3550 \times 10^{-6}$, and $1.0267 \times 10^{-5} \mathrm{~cm}^{-1}$. For ${ }^{32} \mathrm{SO}_{2}-\mathrm{H}_{2}$ the reduced mass is 1.9540708 amu. The symmetry axis ( $z$-axis) corresponds, in standard spectroscopic notation, to the $b$-axis. The nuclear spin statistics for the identical oxygen nuclei with spin 0 allow only asymmetric top levels, $j_{K_{\mathrm{a}} K_{\mathrm{c}}}$, with $K_{\mathrm{a}}, K_{\mathrm{c}}$ either $e e$ or $o o$, where $e / o$ indicates even/odd integer values (Green 1995). The calculated energies of the lowest 31 rotational levels of $\mathrm{SO}_{2}$ are compared in Table 1 to the values given in the JPL catalog (Pickett et al. 1998).

The calculations were carried out using the hybrid logderivative Airy propagator of Alexander \& Manolopoulos (1987). The various propagation parameters were tested to obtain convergence for the lowest 31 levels and for collision energies up to $150 \mathrm{~cm}^{-1}$. Typically the minimum and the maximum integration range was $R_{\min }=5$ and $R_{\max }=40$ bohr. The Airy

\footnotetext{
${ }^{2}$ http://www-laog.obs.ujf-grenoble.fr/valiron/ molscat/index.html
}

Table 1. $\mathrm{SO}_{2}$ calculated energy levels (in $\mathrm{cm}^{-1}$ ) compared to values deduced from the JPL molecular spectroscopy catalog in Pickett et al. (1998).

\begin{tabular}{|c|c|c|c|}
\hline Level & $j_{K_{\mathrm{a}}, K_{\mathrm{c}}}$ & This work & JPL. \\
\hline 1 & $0_{0,0}$ & 0.0000 & 0.0000 \\
\hline 2 & $2_{0,2}$ & 1.9118 & 1.9120 \\
\hline 3 & $1_{1,1}$ & 2.3209 & 2.3208 \\
\hline 4 & $2{ }_{1,1}$ & 3.6973 & 3.6975 \\
\hline 5 & $3_{1,3}$ & 5.3820 & 5.3820 \\
\hline 6 & $4_{0,4}$ & 6.3585 & 6.3601 \\
\hline 7 & $4_{1,3}$ & 8.3327 & 8.3356 \\
\hline 8 & $2_{2,0}$ & 8.7482 & 8.7469 \\
\hline 9 & $3_{2,2}$ & 10.6600 & 10.6590 \\
\hline 10 & $5_{1,5}$ & 10.8856 & 10.8864 \\
\hline 11 & $4_{2,2}$ & 13.2254 & 13.2258 \\
\hline 12 & $6_{0,6}$ & 13.3070 & 13.3134 \\
\hline 13 & $6_{1,5}$ & 15.6009 & 15.6140 \\
\hline 14 & $5_{2,4}$ & 16.3935 & 16.3950 \\
\hline 15 & 7,7 & 18.8219 & 18.8247 \\
\hline 16 & $3_{3,1}$ & 19.2034 & 19.1968 \\
\hline 17 & $6_{2,4}$ & 20.2858 & 20.2941 \\
\hline 18 & $4_{3,1}$ & 21.7560 & 21.7502 \\
\hline 19 & $8_{0,8}$ & 22.7113 & 22.7267 \\
\hline 20 & $7_{2,6}$ & 24.6619 & 24.6713 \\
\hline 21 & $5_{3,3}$ & 24.9465 & 24.9425 \\
\hline 22 & $8_{1,7}$ & 25.4805 & 25.5183 \\
\hline 23 & $6_{3,3}$ & 28.7763 & 28.7760 \\
\hline 24 & $9_{1,9}$ & 29.1782 & 29.1848 \\
\hline 25 & $8_{2,6}$ & 29.9571 & 29.9882 \\
\hline 26 & 73,5 & 33.2419 & 33.2472 \\
\hline 27 & $4_{4,0}$ & 33.7139 & 33.6930 \\
\hline 28 & $10_{0,10}$ & 34.5216 & 34.5495 \\
\hline 29 & $9_{2,8}$ & 35.4513 & 35.4777 \\
\hline 30 & $5_{4,2}$ & 36.9041 & 36.8845 \\
\hline 31 & $10_{1,9}$ & 37.9416 & 38.0265 \\
\hline
\end{tabular}

propagation starts from $R=20$ bohr with an accuracy parameter taken as 0.0003 . We increased the MOLSCAT's parameter STEPS from 10 at the highest energies $\left(\geq 100 \mathrm{~cm}^{-1}\right)$ up to 70 at the lowest energy in order to properly describe the details of the radial function. Our calculations provide state-to-state collisional rate coefficients for the 31 lowest $\mathrm{SO}_{2}$ levels, 72 levels were included in the basis set to obtain convergence better than $5 \%$ on the collisional cross sections. Two calculations for excitation of $\mathrm{SO}_{2}$ by para- $\mathrm{H}_{2}$ were performed, the first one was restricted to $\mathrm{H}_{2}\left(j_{\mathrm{H}_{2}}=j_{2}=0\right)$ and the second one includes in addition the closed $j_{2}=2$ channel. For ortho- $\mathrm{H}_{2}$, the $j_{2}=3$ level of $\mathrm{H}_{2}$ was considered unnecessary at the investigated energies and only the $j_{2}=1$ level was included.

The energy grid, detailed in Table 2 (with $E=E_{\mathrm{c}}+E_{\mathrm{rot}\left(\mathrm{SO}_{2}\right)}$ where $E_{\mathrm{c}}$ is the kinetic energy), was adjusted to reproduce all the details of the resonances as they are essential to calculate the rates with high confidence. Due to the increasingly large computer time and memory ressources needed for the calculations, extension of full close-coupling calculations to higher energies than $150 \mathrm{~cm}^{-1}$ is not realistic. So we adopt an extrapolation procedure for energies from $150 \mathrm{~cm}^{-1}$ up to $300 \mathrm{~cm}^{-1}$ in order to obtain fully converged rate coefficients for temperatures up to $30 \mathrm{~K}$. This extrapolation assumes constant values for the cross sections from the last calculated value $\left(150 \mathrm{~cm}^{-1}\right)$. Several controls of this approximation were performed, including evaluation of the contribution of the extrapolated part of the cross sections as well as check of the detailed balance between upwards and downwards rates. At low temperatures, the effect 
Table 2. Energy grid of the quantum MOLSCAT cross section calculations.

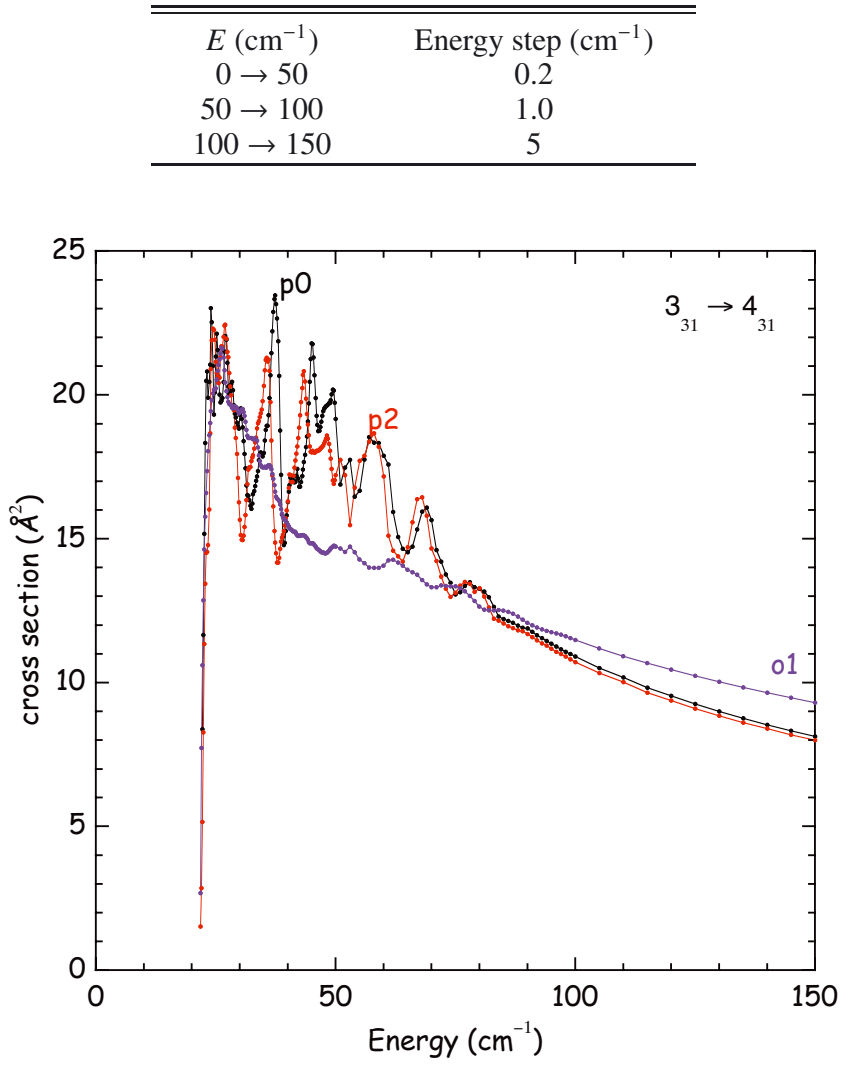

Fig. 1. Cross section for excitation by para and ortho- $\mathrm{H}_{2}$ for the $3_{31} \rightarrow 4_{31}$ transition as a function of the energy $E=E_{\mathrm{c}}+E_{\mathrm{rot}\left(\mathrm{SO}_{2}\right)}$. Black curve: para- $\mathrm{H}_{2}\left(j_{2}=0\right)$; red curve para- $\mathrm{H}_{2}=\left(j_{2}=0,2\right)$; blue curve: ortho- $\mathrm{H}_{2}\left(j_{2}=1\right)$.

of this extrapolation was found to be negligible and less than one percent at $30 \mathrm{~K}$.

Figures 1 and 2 show cross sections for excitation of $\mathrm{SO}_{2}$ by $\operatorname{para}-\mathrm{H}_{2}\left(j_{2}=0\right)$, para- $\mathrm{H}_{2}\left(j_{2}=0,2\right)$ and ortho- $\mathrm{H}_{2}\left(j_{2}=1\right)$ for transitions $3_{31} \rightarrow 4_{31}$ and $4_{31} \rightarrow 4_{40}$. These figures show that cross-sections for para- $\mathrm{H}_{2}\left(j_{2}=0,2\right)$ and $\left(j_{2}=0\right)$ are almost identical. In particular, these cross-sections display many resonances with similar amplitudes and only a very small shift for some of them. Away from the resonance region, the cross sections vary smoothly with energy. The very small contribution of the $\mathrm{H}_{2}\left(j_{2}=2\right)$ rotational level could be attributed to the high energy of the corresponding closed channels compared to the collisional energy and features of the PES (Spielfiedel et al. 2009).

The situation is different when we compare excitation crosssections by para and ortho- $\mathrm{H}_{2}$. The cross sections differ both by their amplitudes and by the behavior of the resonances, which are much less pronounced for collisions involving ortho- $\mathrm{H}_{2}$. The weaker impact of resonances on collisions with ortho- $\mathrm{H}_{2}$ was also found in $\mathrm{H}_{2} \mathrm{O}-\mathrm{H}_{2}$ (Grosjean et al. 2003) and $\mathrm{HC}_{3} \mathrm{~N}-\mathrm{H}_{2}$ (Wernli et al. 2007a,b) collisions. These differences between collisions with para and ortho- $\mathrm{H}_{2}$ could come from the contribution of additional coupling terms (in particular to the dipolequadrupole term) absent in collisions with para- $\mathrm{H}_{2}$.

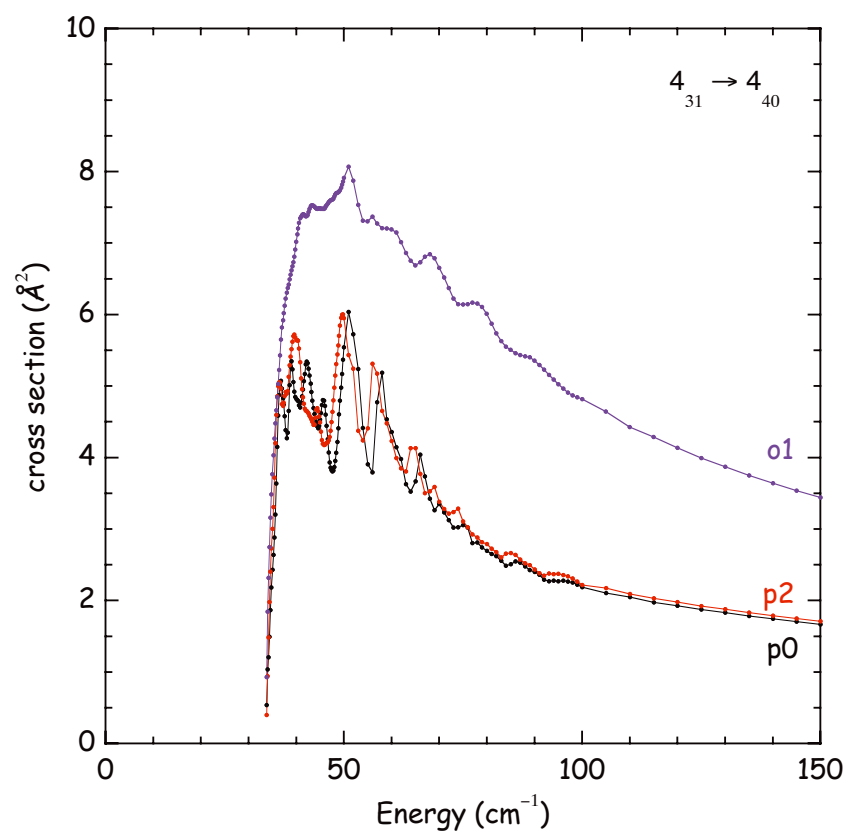

Fig. 2. Cross section for excitation by para and ortho- $\mathrm{H}_{2}$ for the $4_{31} \rightarrow 4_{40}$ transition as a function of the energy $E=E_{\mathrm{c}}+E_{\mathrm{rot}\left(\mathrm{SO}_{2}\right)}$. Black curve: para- $\mathrm{H}_{2}\left(j_{2}=0\right)$; red curve para- $\mathrm{H}_{2}=\left(j_{2}=0,2\right)$; blue curve: ortho- $\mathrm{H}_{2}\left(j_{2}=1\right)$.

\subsection{Para and ortho $\mathrm{H}_{2}$ rates}

In the considered energy range, $\mathrm{H}_{2}$ is always in states $j_{2}=0$ and $j_{2}=1$ for para and ortho $\mathrm{H}_{2}$, respectively, so we drop in the following the $j_{2}$ index. The state-to-state rotational inelastic rates are the Boltzmann thermal average at temperature $T$ of state-to-state inelastic cross sections:

$$
\begin{aligned}
R\left(\beta \rightarrow \beta^{\prime}\right)= & \left(\frac{8 k T}{\pi \mu}\right)^{\frac{1}{2}}\left(\frac{1}{k T}\right)^{2} \\
& \times \int_{0}^{\infty} \sigma_{\beta \rightarrow \beta^{\prime}}\left(E_{\mathrm{c}}\right) E_{\mathrm{c}} \mathrm{e}^{-E_{\mathrm{c}} / k T} \mathrm{~d} E_{\mathrm{c}}
\end{aligned}
$$

where $k$ is the Boltzmann constant, $E_{\mathrm{c}}$ the kinetic energy, and $\beta=j_{K \mathrm{a}, K \mathrm{c}}$. Rate coefficients obey the detailed balance relation:

$R\left(\beta^{\prime} \rightarrow \beta\right)=\frac{(2 j+1)}{\left(2 j^{\prime}+1\right)} \exp \left[\frac{E_{\beta^{\prime}}-E_{\beta}}{k T}\right] R\left(\beta \rightarrow \beta^{\prime}\right)$.

We compare in Table 3 a sample of the present collisional rates at $25 \mathrm{~K}$ with those of Green (1995). It should be noted that the He rates of Green (1995) have been scaled by the reduced mass ratio of $\left(\mu_{\mathrm{SO}_{2}-\mathrm{H}_{2}} / \mu_{\mathrm{SO}_{2}-\mathrm{He}}\right)^{1 / 2}=1.39$. We first note significant discrepancies between the present rates and the scaled He-rates, even for collisions with para- $\mathrm{H}_{2}(j=0)$. Differences do not vary uniformly with transitions and are found to exceed a factor of ten in some cases. These discrepancies are expected because the rates were computed by Green within the IOS scattering approach based on a coarse electron-gas approximation used for the PES. However, we point out that similar trends for $\mathrm{He} / \mathrm{H}_{2}$ have been found for other systems such as $\mathrm{SO}$ (Lique et al. 2007), SiS (Lique et al. 2008), $\mathrm{HC}_{3} \mathrm{~N}$ (Wernli et al. 2007a,b), and $\mathrm{H}_{2} \mathrm{CO}$ (Troscompt et al. 2009), even though the PES's and the cross sections were computed with the same accuracy. For all these systems, the potential well for interaction with $\mathrm{H}_{2}$ is deeper by a factor of about two than the one found for interaction with $\mathrm{He}$, leading to larger cross sections and rates for 
Table 3. Rate coefficients (in $\mathrm{cm}^{3} \mathrm{~s}^{-1}$ ) for the rotational deexcitation of $\mathrm{SO}_{2}$ by collisions with $\mathrm{He}\left(\right.$ Green 1995 , scaled), para- $\mathrm{H}_{2}\left(j_{2}=0\right)$, para- $\mathrm{H}_{2}$ $\left(j_{2}=0,2\right)$, ortho- $\mathrm{H}_{2}\left(j_{2}=1\right)$ at $T=25 \mathrm{~K}^{a}$.

\begin{tabular}{|c|c|c|c|c|c|}
\hline \multicolumn{2}{|c|}{ States of $\mathrm{SO}_{2}$} & \multirow[b]{2}{*}{ He (scaled) } & \multirow{2}{*}{$\begin{array}{c}\text { This work } \\
\text { para- } \mathrm{H}_{2}\left(j_{2}=0\right)\end{array}$} & \multirow{2}{*}{$\begin{array}{c}\text { This work } \\
\text { para- } \mathrm{H}_{2}\left(j_{2}=0,2\right)\end{array}$} & \multirow{2}{*}{$\begin{array}{c}\text { This work } \\
\text { ortho- } \mathrm{H}_{2}\left(j_{2}=1\right)\end{array}$} \\
\hline initial & final & & & & \\
\hline 202 & $0_{00}$ & $1.16(-11)$ & $4.055(-11)$ & $3.981(-11)$ & $3.827(-11)$ \\
\hline $1_{11}$ & $0_{00}$ & $3.98(-12)$ & $1.723(-11)$ & $1.666(-11)$ & $4.253(-11)$ \\
\hline $22_{11}$ & $0_{00}$ & 0 & $3.039(-12)$ & $3.404(-12)$ & $1.562(-11)$ \\
\hline $3_{13}$ & $0_{00}$ & $1.90(-12)$ & $1.567(-11)$ & $1.595(-11)$ & $1.211(-11)$ \\
\hline $4_{04}$ & $0_{00}$ & $1.43(-12)$ & $7.905(-12)$ & $8.604(-12)$ & $8.004(-12)$ \\
\hline $4_{13}$ & $0_{00}$ & 0 & $1.212(-12)$ & $1.232(-12)$ & $4.514(-12)$ \\
\hline 220 & $0_{00}$ & $1.63(-12)$ & $1.851(-11)$ & $2.008(-11)$ & $1.504(-11)$ \\
\hline $3_{22}$ & $0_{00}$ & 0 & $1.967(-12)$ & $2.402(-12)$ & $4.903(-12)$ \\
\hline $5_{15}$ & $0_{00}$ & $1.13(-12)$ & $7.223(-12)$ & $7.357(-12)$ & $5.817(-12)$ \\
\hline
\end{tabular}

Notes. ${ }^{(a)}$ Powers of ten are given in parenthesis.

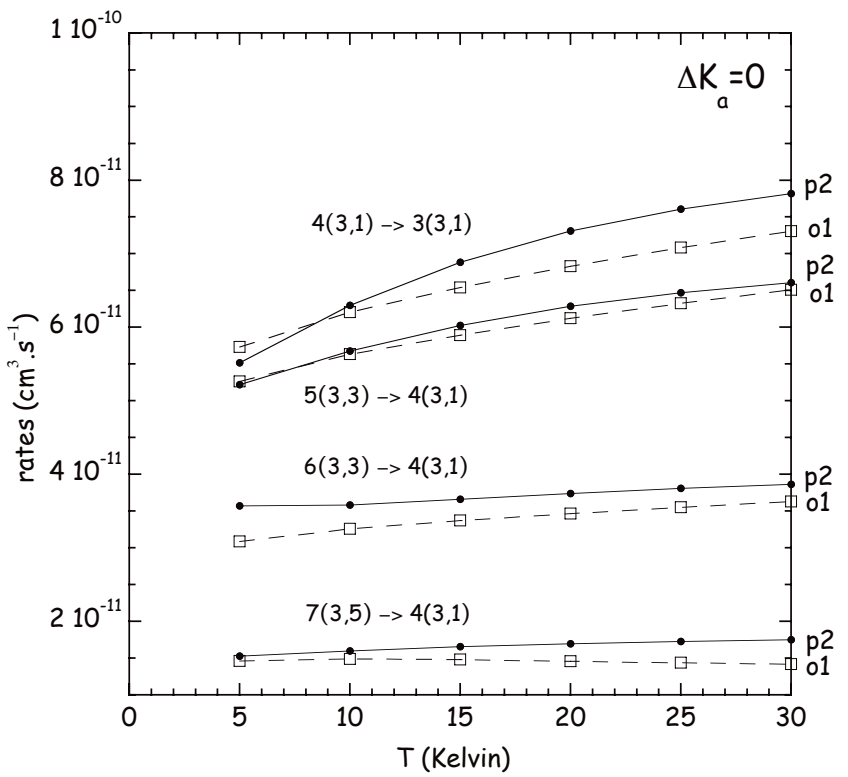

Fig. 3. Temperature variation of the de-excitation rate coefficient for transitions with $\Delta K_{\mathrm{a}}=0 . \mathrm{p} 2$ and $\mathrm{o} 1$ refer to para and ortho- $\mathrm{H}_{2}$ rate coefficients.

collisions with $\mathrm{H}_{2}$. Moreover, preliminary rate coefficients obtained in the IOS approximation with the same $\mathrm{SO}_{2}-\mathrm{H}_{2} \mathrm{PES}$ as in the present calculations are systematically twice/three times less than the $\mathrm{CC}$ results. All these factors contribute to the large underestimation of the scaled He-rates of Green for collisions with $\mathrm{H}_{2}$.

Figures 3 and 4 display the temperature variation of the para and ortho- $\mathrm{H}_{2}$ rate coefficients for a selection of $\Delta K_{\mathrm{a}}=0$ (Fig. 3) and $\Delta J=0$ (Fig. 4) transitions. The following trends are found: the temperature dependence is weak within factor two to three for both para and ortho- $\mathrm{H}_{2}$ collisions. There is no exact selection rule, but the rates decrease for increasing $|\Delta J|$ and $\left|\Delta K_{\mathrm{a}}\right|$. This propensity is pointed out in Fig. 5, which presents the excitation/deexcitation rates at $25 \mathrm{~K}$ for collisions with para- $\mathrm{H}_{2}$ from level $J_{K_{\mathrm{a}} K_{\mathrm{c}}}=4_{31}$. One can observe that the most important rates are those that connect levels with $\left|\Delta K_{\mathrm{a}}\right|=0$. This trend may be explained by the difficulty of reorienting the angular momentum vector with respect to the $a$-axis.

Figure 6 compares the de-excitation rate coefficients at $25 \mathrm{~K}$ for collisions of $\mathrm{SO}_{2}$ with para- $\mathrm{H}_{2}\left(j_{2}=0\right)$ and ortho- $\mathrm{H}_{2}$ with rate coefficients for collisions with para- $\mathrm{H}_{2}\left(j_{2}=0,2\right)$. It is seen that including of $\mathrm{H}_{2}\left(j_{2}\right)=2$ has a negligible contribution

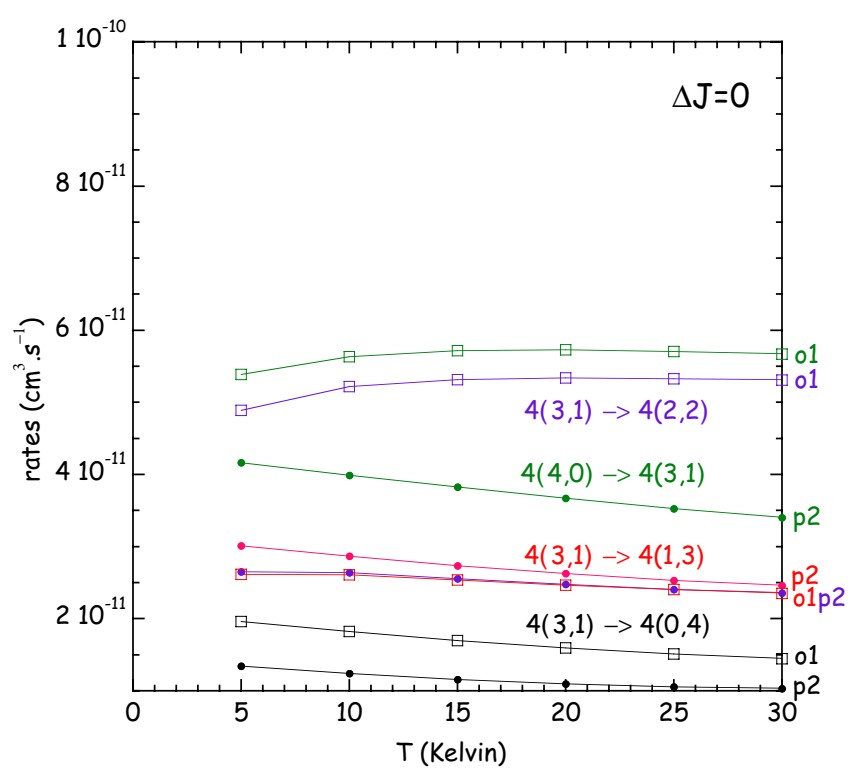

Fig. 4. Temperature variation of the de-excitation rate coefficient for transitions with $\Delta J=0 . \mathrm{p} 2$ and o1 refer to para and ortho- $\mathrm{H}_{2}$ rate coefficients.

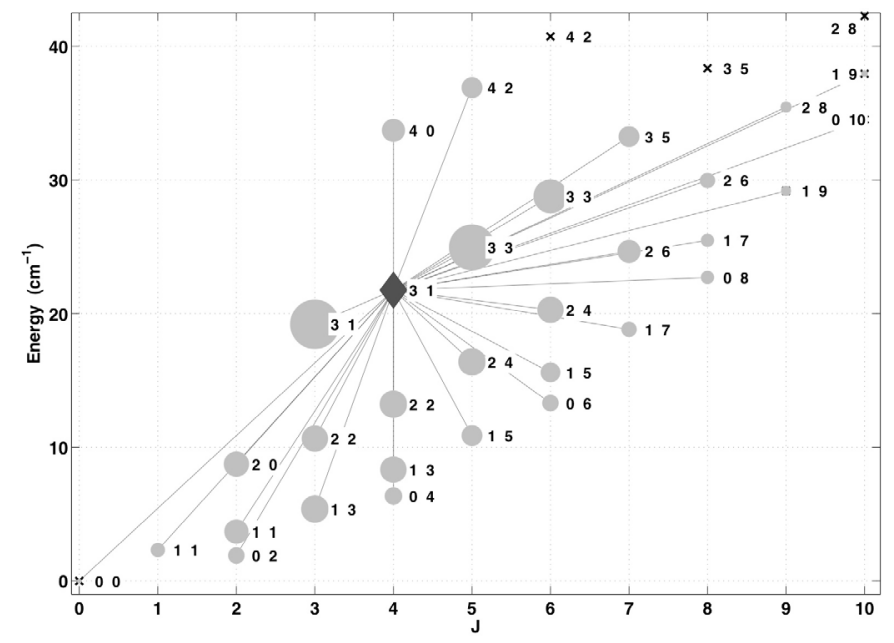

Fig. 5. $\mathrm{SO}_{2}$ rotational levels connected to the initial one $\left(4_{31}\right)$, denoted by a black diamond. Those marked by a gray disk have a para- $\mathrm{H}_{2}$ collisonal excitation or deexcitation rate above the threshold $>10^{-12} \mathrm{~cm}^{3} \mathrm{~s}^{-1}$, with the area of each gray disk proportional to the rate. Crosses correspond to rates below the threshold. 

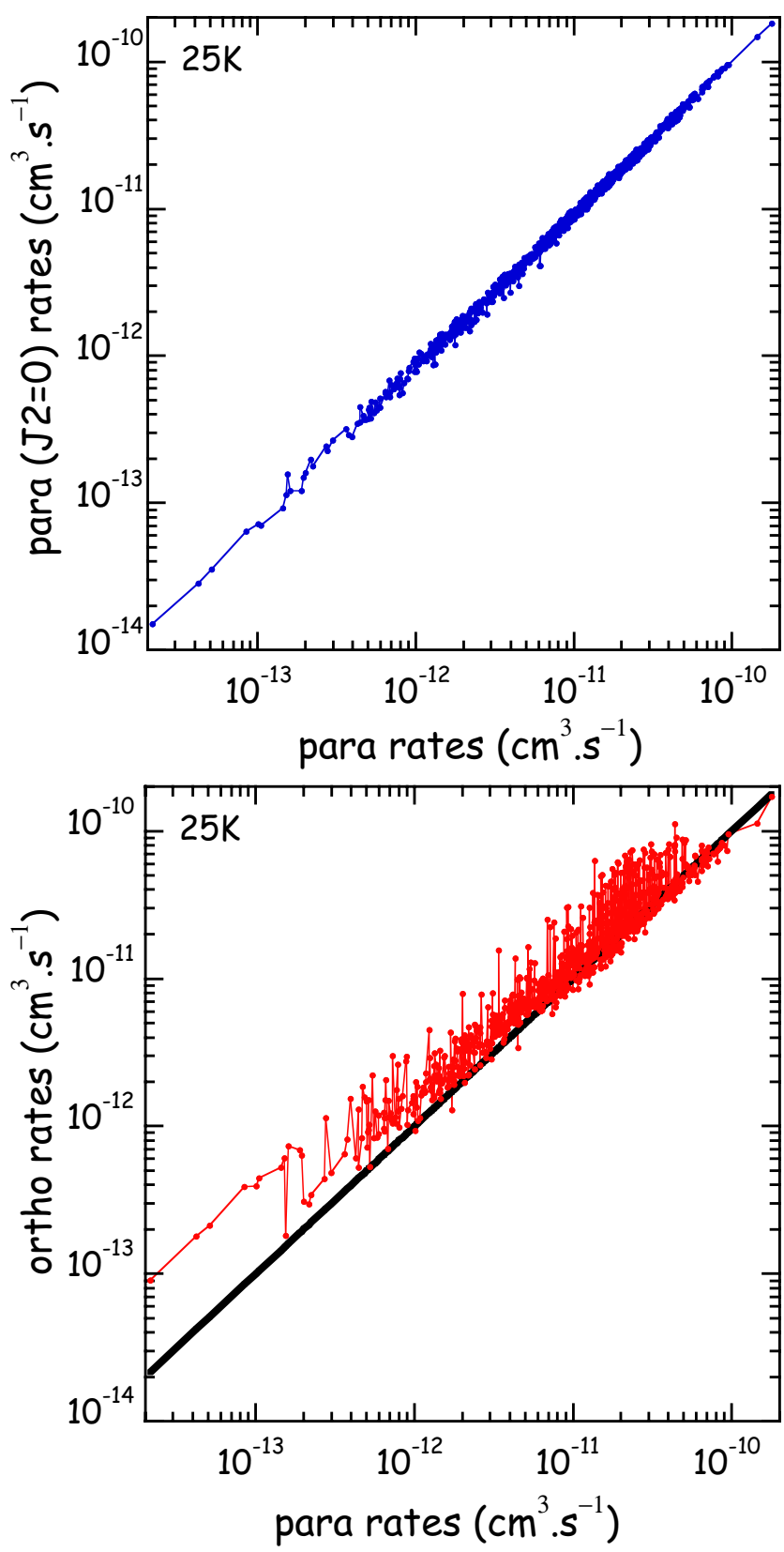

Fig. 6. De-excitation rate coefficients of $\mathrm{SO}_{2}$ in collision with para$\mathrm{H}_{2}\left(j_{2}=0\right)$ as function of rate coefficients with para- $\mathrm{H}_{2}\left(j_{2}=0,2\right)$. The black line indicates equal rates.

to para- $\mathrm{H}_{2}$ rate coefficients, and that both sets of rates agree within a few percent. Rate coefficients with ortho and para- $\mathrm{H}_{2}$ vary strongly with the transition, with a factor of about two on average, the largest rate coefficients being mainly the rates for collisions with ortho- $\mathrm{H}_{2}$. The latter effect reflects the importance of the $\mathrm{H}_{2}$ quadrupole and in particular the dipolequadrupole interaction, which does not contribute to collisions with para- $\mathrm{H}_{2}\left(j_{2}=0\right)$.

All rates among the 31 first rotational levels are provided as online material for a temperature range $5 \mathrm{~K} \leq T \leq 30 \mathrm{~K}$ (Tables 5 and 6). They will be made available in the LAMDA ${ }^{3}$ and BASECOL ${ }^{4}$ databases.

\footnotetext{
${ }^{3}$ http://www.strw.leidenuniv.nl/ moldata/

${ }^{4}$ http://basecol.obspm.fr/
}

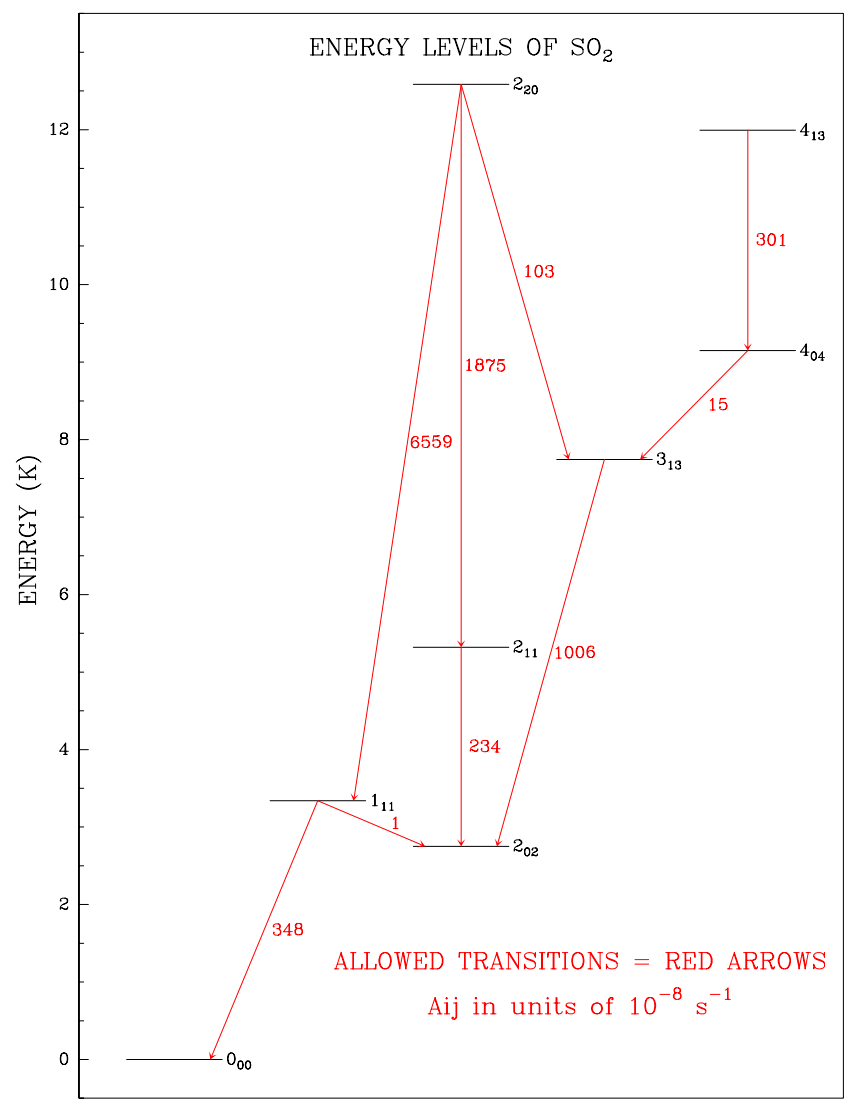

Fig. 7. Rotational levels of $\mathrm{SO}_{2}$ with energies below $13 \mathrm{~K}$. Allowed radiative transitions are indicated by red arrows. The numbers indicate the corresponding Einstein coefficients for spontaneous emission in units of $10^{-8} \mathrm{~s}^{-1}$.

\section{3. $\mathrm{SO}_{2}$ line absorption and maser emission in dark clouds}

\subsection{Centimeter wavelength transitions}

$\mathrm{SO}_{2}$ was detected in interstellar space in the early years of millimeter radioastronomy (Snyder et al. 1975). To our knowledge, all studies of interstellar and circumstellar clouds using $\mathrm{SO}_{2}$ have been performed in the millimeter and submillimeter domains, i.e., by observing rotational transitions involving high energy levels. However, as a heavy species, $\mathrm{SO}_{2}$ has several transitions in the centimeter domain arising from low-lying levels (see Fig. 7). The structure of these levels makes it certain that $\mathrm{SO}_{2}$ should show strong excitation effects in cold dark clouds, that have so far not been analyzed.

Owing to the $\mathrm{C}_{2 v}$ symmetry of the molecule, the first excited rotational level, $2_{02}$ at $2.75 \mathrm{~K}$, is not radiatively connected to the ground level, $0_{00}$. Consequently, $2_{02}$ has to be considered as a metastable level. Under non-LTE conditions, e.g. for the physical conditions of dark clouds, it will be overpopulated with respect to the closest level, the $1_{11}$, at $3.34 \mathrm{~K}$ which is radiatively connected to the ground level. Hence, the rotational transition $1_{11}-2_{02}$ at $12256.583 \mathrm{MHz}$ could be in absorption against the cosmic microwave background for a considerably wide range of densities and temperatures. The Einstein coefficient for the transition $1_{11}-0_{00}$ is $\left(3.5 \times 10^{-6} \mathrm{~s}^{-1}\right)$, so that high volume densities will be required in order to thermalize the lowest energy levels of $\mathrm{SO}_{2}$, including the metastable one $2_{02}$.

A second group of rotational levels, $4_{13}, 4_{04}$ and 313 around $12,9.1$, and $7.7 \mathrm{~K}$, respectively, will also show peculiar 


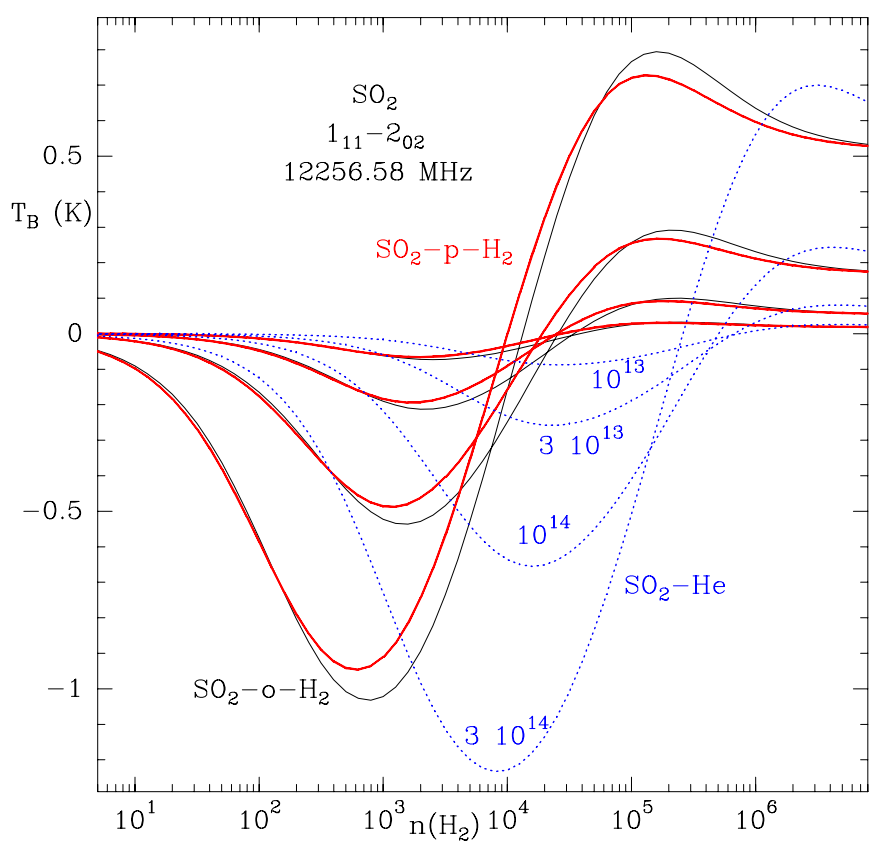

Fig. 8. Predicted brightness temperature for the $1_{11}-2_{02}$ line of $\mathrm{SO}_{2}$ at $12.256 \mathrm{GHz}$ for different sets of collisional rates. The assumed kinetic temperature and $\Delta v=$ are $10 \mathrm{~K}$ and $1 \mathrm{~km} \mathrm{~s}^{-1}$, respectively. Black(thin)/red(thick) lines correspond to the excitation of the molecule by $\mathrm{o}-\mathrm{H}_{2} / \mathrm{p}-\mathrm{H}_{2}$ using the collisional rates calculated in this work. Blue(dashed) lines correspond to the predicted intensity when the scaled He-rates of Green (1995) are used. Column densities are indicated and correspond to $3 \times 10^{14}, 10^{14}, 3 \times 10^{13}$, and $10^{13} \mathrm{~cm}^{-2}$.

excitation conditions. The $4_{13}$ level will radiatively decay towards the $4_{04}$ one (only possible transition arising from the $4_{13}$ level; its Einstein coefficient is $3 \times 10^{-6} \mathrm{~s}^{-1}$ ), which will slowly decay towards the $3_{13}$ level. The latter will decay very fast towards the metastable level $2_{02}$ (see the Einstein coefficients in Fig. 7). The consequence is that the $4_{04}$ and $3_{13}$ levels could be inverted; i.e., the transition $4_{04}-3_{13}$ at $29321.331 \mathrm{MHz}$ could be maser in nature. Although these effects mainly come from the structure of the energy levels and the Einstein coefficients of the rotational transitions, collisions of $\mathrm{SO}_{2}$ with $\mathrm{H}_{2}$ will play an important role in quenching the maser and the absorption lines for some critical densities. In order to quantify these effects, we performed LVG excitation calculations using our new, state of the art, collisional rates for para and ortho $\mathrm{H}_{2}$. The results of our excitation calculations are shown in Figs. 8 and 9 for a kinetic temperature of $10 \mathrm{~K}$ and indicate that our qualitative analysis was correct: the $1_{11}-2_{02}$ transition will be in absorption and the $4_{04}-3_{13}$ will be maser for a wide range value of volume densities, temperatures, and abundances. For comparison purposes, the results obtained using the collisional rates calculated by Green (1995) for the $\mathrm{SO}_{2}-\mathrm{He}$ system, scaled by the factor 1.39, are also shown in Figs. 8 and 9. While rotational excitation by ortho and para molecular hydrogen produce similar results, collisions with helium using the rates of Green (1995), corrected by a factor 1.39 to take the different reduced mass of $\mathrm{SO}_{2} / \mathrm{He}$ and $\mathrm{SO}_{2} / \mathrm{H}_{2}$ into account, produces a shift of the curves to higher densities (a factor 10). As discussed in previous sections, this effect is due to the higher $\mathrm{SO}_{2}-(\mathrm{o} / \mathrm{p})-\mathrm{H}_{2}$ collisional rates (one order of magnitude) than those of the $\mathrm{SO}_{2}-\mathrm{He}$ system. This effect has been found for all rotational transitions of $\mathrm{SO}_{2}$ arising from the 31 energy levels considered in this paper (see below). This large difference in density between molecular

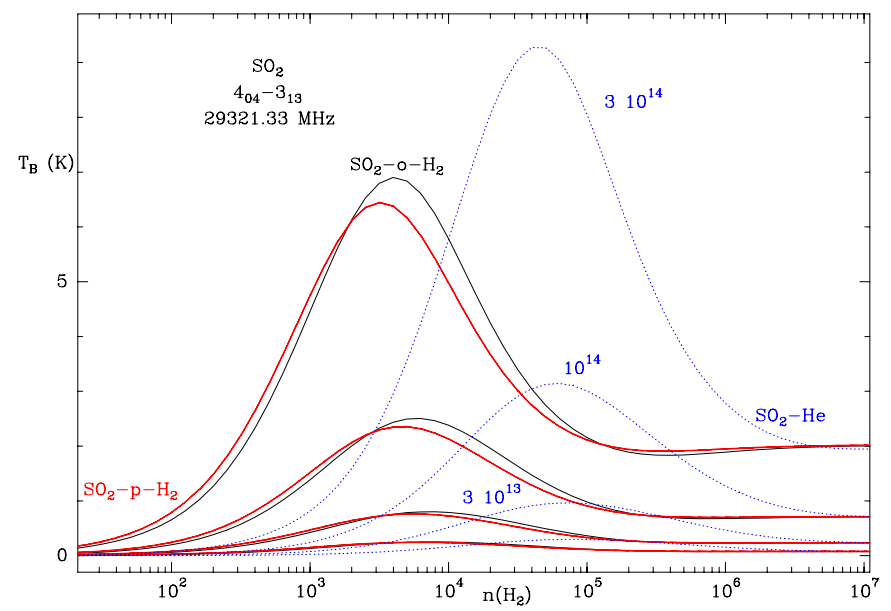

Fig. 9. Predicted brightness temperature for the $4_{04}-3_{13}$ line of $\mathrm{SO}_{2}$ at $29.321 \mathrm{GHz}$ for different sets of collisional rates. The assumed kinetic temperature and $\Delta v=$ are $10 \mathrm{~K}$ and $1 \mathrm{~km} \mathrm{~s}^{-1}$, respectively. Black(thin)/red(thick) lines correspond to the excitation of the molecule by $\mathrm{o}-\mathrm{H}_{2} / \mathrm{p}-\mathrm{H}_{2}$ using the collisional rates calculated in this work. Blue dotted lines correspond to the predicted intensity when the rates of Green (1995) for the $\mathrm{SO}_{2}-\mathrm{He}$ system are used. Column densities are indicated and correspond to $3 \times 10^{14}, 10^{14}, 3 \times 10^{13}$, and $10^{13} \mathrm{~cm}^{-2}$.

hydrogen and helium rates has deep implications for determining $\mathrm{SO}_{2}$ abundances in cold and warm molecular clouds. The new rates indicate that $\mathrm{SO}_{2}$ rotational levels will be thermalized for densities 10-20 times lower than those predicted from Green's rates. As a result, $\mathrm{SO}_{2}$ seems more easily thermalized than previously thought. As a consequence, the derived column densities (and abundances) in previous works are probably too high.

Our calculations indicate that, for a density of $3 \times 10^{3} \mathrm{~cm}^{-3}$, a kinetic temperature of $10 \mathrm{~K}$ (rates for $\mathrm{SO}_{2}-\mathrm{He}$ have been extrapolated down to $10 \mathrm{~K}$ using a square root dependence on the temperature), a linewidth of $1 \mathrm{~km} \mathrm{~s}^{-1}$, and a column density above $3 \times 10^{12} \mathrm{~cm}^{-2}$ (which could correspond to an abundance of $3 \times 10^{-10}$ for a cloud of 10 mag of visual absorption), the maser line will have an intensity higher than $0.07 \mathrm{~K}$. The $\mathrm{SO}_{2}$ abundances derived in dark clouds (Irvine et al. 1983; Dickens et al. 2000) range from 2 to $6 \times 10^{-9}$, so the $4_{04}-3_{13}$ line could have brightness temperatures above $3 \mathrm{~K}$ in most cases. Moreover, the maser line will be the strongest transition of $\mathrm{SO}_{2}$ in cold dark clouds. This is an important issue because the millimeter lines of this species (Snyder et al. 1975; Irvine et al. 1983) are rather weak and have only been observed towards a few selected dense cold cores. Finally, for the range of abundances found for $\mathrm{SO}_{2}$ in dark clouds, the $1_{11}-2_{02}$ absorption line will be as strong as $-0.5 \mathrm{~K}$ for these volume densities. Even for column densities as low as $3 \times 10^{12} \mathrm{~cm}^{-2}$, the absorption that could be observed in this line against the cosmic background will be $\simeq-0.02 \mathrm{~K}$.

We checked that the absorption and masering effects do not depend on the selected temperature within the range of validity of our collisional rates, 5-30 K. In particular, we have verified that the factor 10 in density between our collisional rates and those of Green is found not only for $10 \mathrm{~K}$ but also for $25 \mathrm{~K}$, the lowest temperature of Green's calculations (i.e., this factor 10 is not produced by the selected square root extrapolation of Green's rates from $25 \mathrm{~K}$ to $10 \mathrm{~K}$ ). 


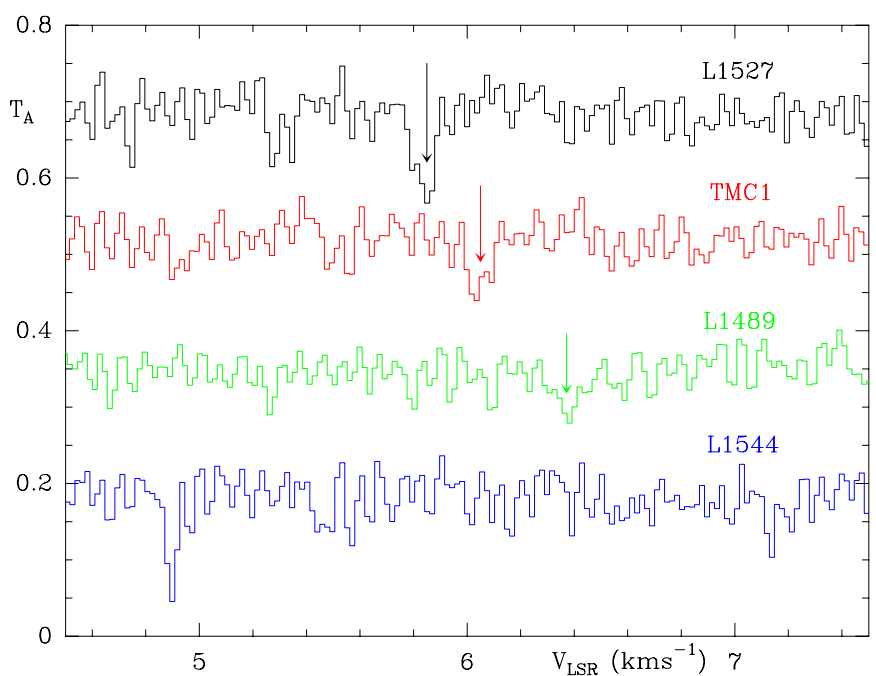

Fig. 10. Raw data for the $1_{11}-2_{02}$ line of $\mathrm{SO}_{2}$ obtained towards four dark clouds. Although the data suffer from a high-frequency ripple plus spurious features produced by human-made interferences, the absorption features appear clearly in three of them, TMC1, L1527, and L1489. Processed data are shown in Fig. 11.

\subsection{Observation of absorption of $\mathrm{SO}_{2}$ against the cosmic background}

In spite of their potential for tracing the physical conditions of cold clouds, none of these lines have ever been observed in dark clouds. The $1_{11}-2_{02}$ line is particularly interesting because the new collisional rates indicate that the line will move from absorption to emission for $n\left(\mathrm{H}_{2}\right)>10^{4} \mathrm{~cm}^{-3}$. The absorption line will therefore be a good tracer of the physical properties of the low-density envelopes of dense cores. Moreover, this line could be used to trace the chemistry of sulfur-bearing molecules in low-density regions (CS, SO, and the millimeter lines of $\mathrm{SO}_{2}$ require high $n\left(\mathrm{H}_{2}\right)$ values to produce detectable emission). The maser $4_{04}-3_{13}$ line will also be particularly interesting for this purpose.

To check our predictions, we observed four dark clouds in 2009 with the GBT telescope at the frequency of the $1_{11}-2_{02}$ line $(12.256 \mathrm{GHz})$. The selected clouds are TMC1 $\left(\alpha_{2000}=04^{\mathrm{h}} 41^{\mathrm{m}} 45.9^{\mathrm{s}}, \delta_{2000}=25^{\circ} 41^{\prime} 26.8^{\prime \prime}\right)$, L1527 $\left(\alpha_{2000}=\right.$ $\left.04^{\mathrm{h}} 39^{\mathrm{m}} 53.0^{\mathrm{s}}, \delta_{2000}=25^{\circ} 45^{\prime} 0.0^{\prime \prime}\right), \mathrm{L} 1544\left(\alpha_{2000}=05^{\mathrm{h}} 04^{\mathrm{m}} 18.1^{\mathrm{s}}\right.$, $\left.\delta_{2000}=25^{\circ} 11^{\prime} 7.9^{\prime \prime}\right)$, and L1489 $\left(\alpha_{2000}=04^{\mathrm{h}} 04^{\mathrm{m}} 47.5^{\mathrm{s}}, \delta_{2000}=\right.$ $\left.26^{\circ} 19^{\prime} 42 \cdot 1^{\prime \prime}\right)$. Abundant observational data, in several molecular species, is available in the literature for these clouds (Cernicharo \& Guélin 1987; Cernicharo et al. 1984; Daniel et al. 2007; Duvert et al. 1986; Fuller et al. 1996; Hogerheijde et al. 1997; Hogerheijde 2001; Myers et al. 1995; Ohashi et al. 1997; Takakuwa et al. 2000; Brinch et al. 2007). Except for TMC1 for which the position corresponds to the cyanopolyyne peak, the selected pointing positions for the other sources correspond to the envelope surrounding the dense cores. As indicated by the calculations shown in Fig. 8, the expected absorption will be weak, will even turn into emission, for densities higher than $10^{4} \mathrm{~cm}^{-3}$.

The observations were done in position-switching mode. At the observing frequency the half power beamwidth of the telescope is 67". The spectral resolution was $0.0187 \mathrm{~km} \mathrm{~s}^{-1}$. The raw data are shown in Fig. 10 without any signal processing except the removal of a degree one baseline. These observations were rather delicate as many human-made spurious signals affected the data despite the protection measurements taken for this site. In addition to the spurious features we also found ripples at
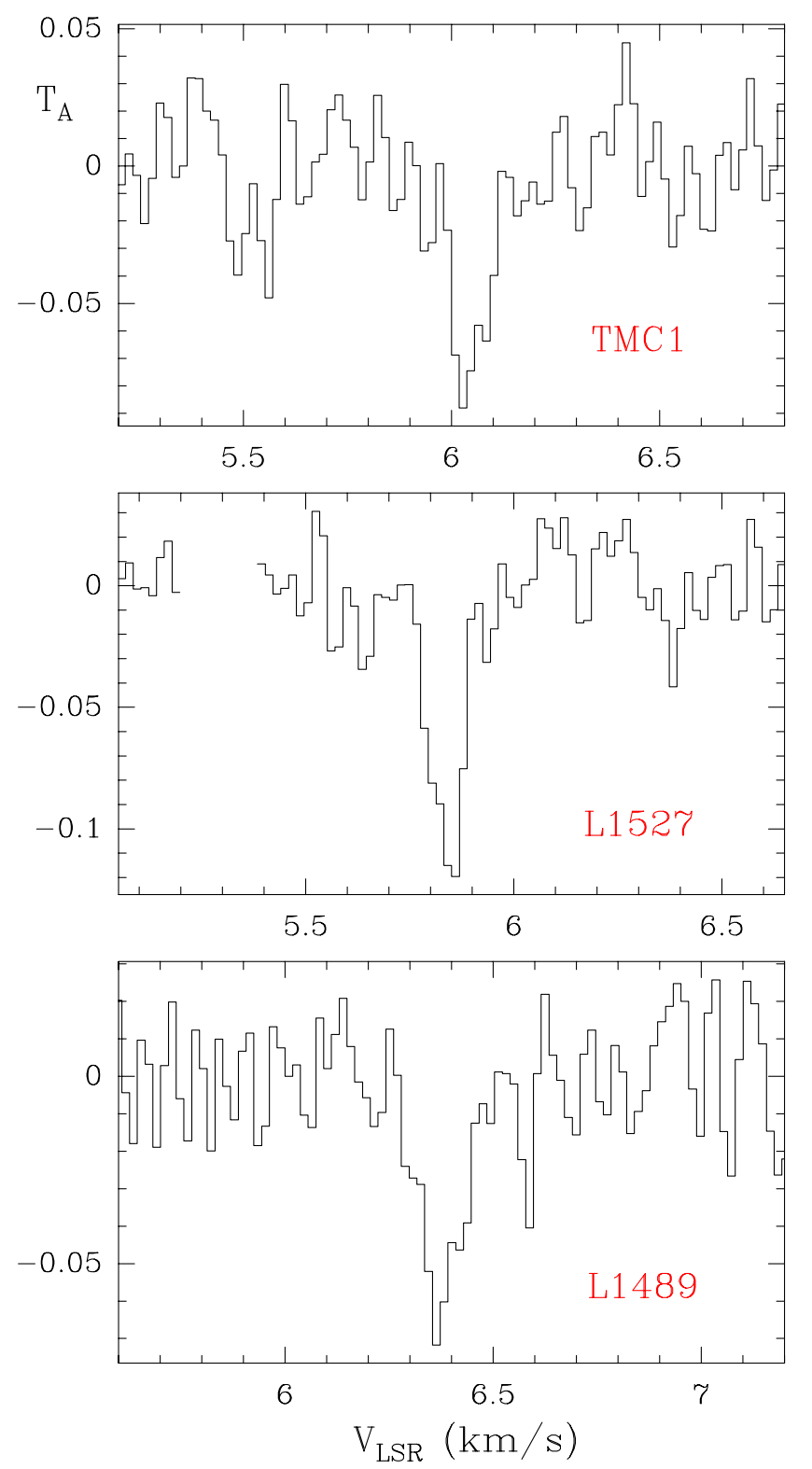

Fig. 11. Observed $1_{11}-2_{02}$ line profile towards TMC1, L1527 and L1489. The raw data without any processing are shown in Fig. 10. The $x$-axis corresponds to LSR velocity and the $y$-axis corresponds to the observed antenna temperature.

different frequencies that were removed by using the GILDAS package. Only a small window around the expected velocity of the clouds, but with enough channels, was finally analyzed for each cloud. The final reduced data are shown in Fig. 11. In spite of the problems found the observations, absorption was observed towards TMC1, L1527, and L1489 at the local standard of rest (LSR) velocity of these clouds. Towards L1544 we obtained only a $3 \sigma$ upper limit of $0.06 \mathrm{~K}$. Figure 11 shows the observed spectra towards TMC1, L1527, and L1489.

The absorption towards TMC1 peaks at $6.04 \mathrm{~km} \mathrm{~s}^{-1}$ with a linewidth of $0.09 \mathrm{~km} \mathrm{~s}^{-1}$ and an intensity of $-0.077 \mathrm{~K}$ (Fig. 11 top panel). This cloud is known to show multiple velocity components (Cernicharo et al. 1984; Cernicharo \& Guélin 1987; Lique et al. 2006). Figure 11 shows a second possible absorption $(3 \sigma)$ at $5.52 \mathrm{~km} \mathrm{~s}^{-1}$ with a linewidth of $0.1 \mathrm{~km} \mathrm{~s}^{-1}$ and an intensity of $-0.038 \mathrm{~K}$. Another sulfur-bearing molecule, SO, has been observed towards this source by Lique et al. (2006), who 
detected two velocity components at 5.6 and $6.0 \mathrm{~km} \mathrm{~s}^{-1}$. The agreement in velocity between the $\mathrm{SO}_{2}$ absorption and the emission found in several lines of SO by Lique et al. (2006) is very good. It suggests that the $\mathrm{SO}_{2}$ absorption arises from the same clouds that emit in the SO lines. Lique et al. (2006) have modeled the gas at $6.0 \mathrm{~km} \mathrm{~s}^{-1}$ with a core of density of $2 \times 10^{4} \mathrm{~cm}^{-3}$ surrounded by a low-density envelope of $6 \times 10^{3} \mathrm{~cm}^{-3}$ and $T_{K}=10 \mathrm{~K}$ for both components. If the observed absorption is arising from the high-density gas, then $N\left(\mathrm{SO}_{2}\right) \simeq 5 \times 10^{12} \mathrm{~cm}^{-2}$. For the assumed volume density the excitation temperature of the $1_{11}-2_{02}$ transition is close to $2.7 \mathrm{~K}$, i.e., the turnover density between absorption and emission for this line (see Fig. 8). However, if the absorption is arising from the low-density envelope, then $N\left(\mathrm{SO}_{2}\right) \simeq 1.2 \times 10^{12} \mathrm{~cm}^{-2}$, so the abundance of $\mathrm{SO}_{2}$ is $\simeq 2 \times 10^{-10}$ (for $A_{\mathrm{v}}=5 \mathrm{mag}$ in the envelope), i.e., two orders of magnitude below that of SO (Lique et al. 2006). The $5.5 \mathrm{~km} \mathrm{~s}^{-1}$ cloud was modeled by Lique et al. (2006) with a single density of $8 \times 10^{3} \mathrm{~cm}^{-3}$. For this component the derived $\mathrm{SO}_{2}$ column density is $6 \times 10^{11} \mathrm{~cm}^{-2}$.

Towards L1527 we selected a position that corresponds to the envelope of the cloud $\left(\Delta \alpha=+3^{\prime}, \Delta \delta=3.5^{\prime}\right.$ with respect HCL2-A Cernicharo et al. 1984; or $\Delta \alpha \simeq+2^{\prime}, \Delta \delta \simeq=7^{\prime}$ from TMC1-A Takakuwa et al. 2000). It is also $30^{\prime \prime}$ east of Elias 18 (Palumbo et al. 2008). The absorption of $\mathrm{SO}_{2}$ towards L1527 peaks at $5.84 \mathrm{~km} \mathrm{~s}^{-1}$ with a linewidth of $0.082 \mathrm{~km} \mathrm{~s}^{-1}$ and an intensity of $-0.11 \mathrm{~K}$ (Fig. 11 central panel). Cernicharo et al. (1984) observed $\mathrm{NH}_{3}$, several lines of $\mathrm{HC}_{3} \mathrm{~N}, \mathrm{HC}_{5} \mathrm{~N}$, and $\mathrm{C}_{4} \mathrm{H}$ towards this cloud (labeled HCL2-A in their paper). They observed velocities between 5.6 and $6.4 \mathrm{~km} \mathrm{~s}^{-1}$ depending on the position. Around the selected one, the averaged velocity is $5.9 \mathrm{~km} \mathrm{~s}^{-1}$. The observed absorption in the $1_{11}-2_{02}$ line of $\mathrm{SO}_{2}$ is too high to be reproduced for a gas with a high density (see Fig. 11). As for all cores in Taurus \& Perseus (Bachiller \& Cernicharo 1986; Cernicharo \& Bachiller 1984; Cernicharo et al. 1985; Cernicharo \& Guélin 1987; Duvert et al. 1986), the core is certainly surrounded by a low-density envelope (see above for TMC1). Assuming a density of $5 \times 10^{3} \mathrm{~cm}^{-3}$, $A_{\mathrm{v}}=3 \mathrm{mag}$, and $T_{K}=10 \mathrm{~K}$, the observed absorption can be reproduced with $N\left(\mathrm{SO}_{2}\right) \simeq 1.5 \times 10^{12} \mathrm{~cm}^{-2}$, which corresponds to $X\left(\mathrm{SO}_{2}\right)=5 \times 10^{-10}$.

For L1489 we selected a position that is at $\Delta \alpha=+1^{\prime}$, $\Delta \delta=+46^{\prime \prime}$ with respect to the IRS1 source (see, e.g., Hogerheijde et al. 1997). Depending on the chosen molecular line the velocity of the gas covers the range $5.5-7 \mathrm{~km} \mathrm{~s}^{-1}$ with most of them peaking around $6.5 \mathrm{~km} \mathrm{~s}^{-1}$. In this cloud we observed an absorption feature at $6.4 \mathrm{~km} \mathrm{~s}^{-1}$ with a linewidth of $0.13 \mathrm{~km} \mathrm{~s}^{-1}$ and an intensity of $-0.07 \mathrm{~K}$. Assuming a density in the envelope of $5 \times 10^{3} \mathrm{~cm}^{-3}$ the observed parameters correspond to a column density for $\mathrm{SO}_{2}$ of $1.5 \times 10^{12} \mathrm{~cm}^{-2}$ and an abundance, assuming $A_{\mathrm{v}} \simeq 3 \mathrm{mag}, X\left(\mathrm{SO}_{2}\right)=5 \times 10^{-10}$.

Finally, the line was not detected towards L1544. Assuming similar physical conditions than for L1489 we derive $N\left(\mathrm{SO}_{2}\right)<$ $10^{12} \mathrm{~cm}^{-2}(3 \sigma)$.

\section{3. $\mathrm{SO}_{2}$ rotational lines in the millimeter domain}

We used the new collisional rates to explore the behavior of the millimeter lines of $\mathrm{SO}_{2}$ that could be detected in cold dark clouds. The calculations were done using the LVG approximation, and the results are shown in Figs. 12 and 13. We selected a kinetic temperature of $10 \mathrm{~K}$, typical of cold dark clouds, and we varied the density between $10^{2}$ and $10^{8} \mathrm{~cm}^{-3}$ for three different column densities of $10^{12}, 10^{13}$, and $10^{14} \mathrm{~cm}^{-2}$, which could correspond to abundances of $10^{-8}$ to $10^{-10}$ for a cloud with

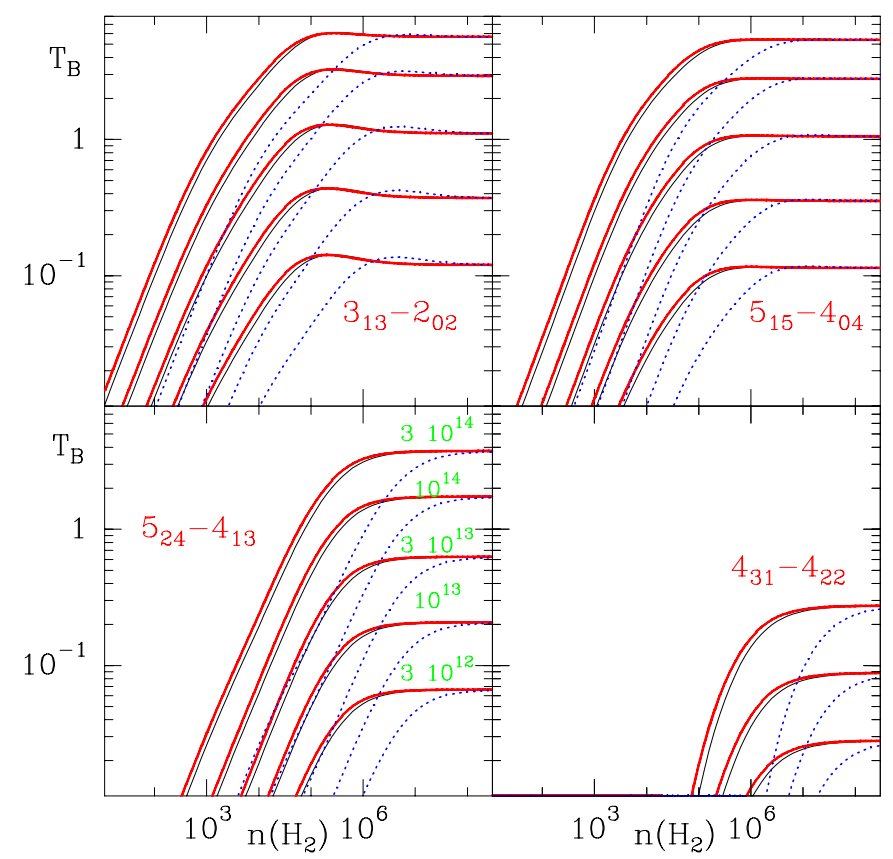

Fig. 12. Predicted intensities for the $\mathrm{SO}_{2}$ lines of Table 4. The assumed kinetic temperature and $\Delta v=$ are $10 \mathrm{~K}$ and $1 \mathrm{~km} \mathrm{~s}^{-1}$, respectively. Units for the density and $T_{B}$ are $\mathrm{cm}^{-3}$ and Kelvin, respectively. Black thin lines correspond to the predictions for the $\mathrm{SO}_{2} / \mathrm{o}-\mathrm{H}_{2}$ system (this work), red thick lines to the $\mathrm{SO}_{2} / \mathrm{p}-\mathrm{H}_{2}$ system (this work), and blue dotted lines to $\mathrm{SO}_{2}-\mathrm{He}$ (rates from Green 1995; extrapolated down to $10 \mathrm{~K}$ as indicated in the text).

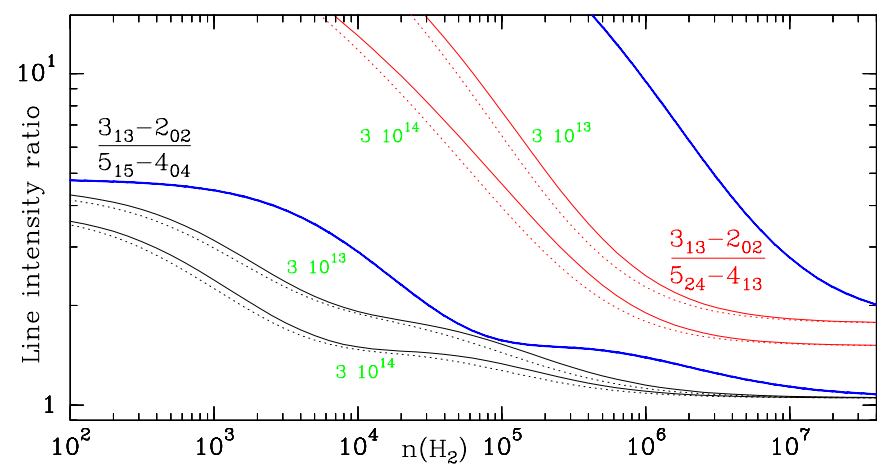

Fig. 13. Predicted line intensity ratios for selected lines of interest for the study of dark clouds. The assumed kinetic temperature and $\Delta v=$ are $10 \mathrm{~K}$ and $1 \mathrm{~km} \mathrm{~s}^{-1}$, respectively. Solid/dashed lines correspond to the case of collisional excitation by ortho/para $\mathrm{H}_{2}$. The results correspond to $N\left(\mathrm{SO}_{2}\right)=3 \times 10^{13}$ and $3 \times 10^{14} \mathrm{~cm}^{-2}$. The blue thick curve corresponds to $N\left(\mathrm{SO}_{2}\right)=3 \times 10^{14} \mathrm{~cm}^{-2}$ but using the collisional rates $\mathrm{SO}_{2} / \mathrm{He}$ of Green (1995).

$N\left(\mathrm{H}_{2}\right)=10^{22} \mathrm{~cm}^{-2}$. Among the lines that could be detected in dark clouds in the millimeter domain, we selected those indicated in Table 4.

The $3_{13}-2_{02}$ line at $104.029 \mathrm{GHz}$ is the strongest one, and the results show that it thermalized for a density of $10^{5} \mathrm{~cm}^{-3}$ (see Fig. 12). The $5_{15}-4_{04}$ and $5_{24}-4_{13}$ lines are thermalized for a density around $10^{6} \mathrm{~cm}^{-3}$. Like the case for the centimeter wavelength transitions, the predictions are practically the same for ortho and para $\mathrm{H}_{2}$. However, if we use the scaled He-rates, these densities have to be multiplied by a factor 10 . The $4_{31}-4_{22}$ line is the weakest of the four lines. Although also thermalized for $n\left(\mathrm{H}_{2}\right)=10^{6} \mathrm{~cm}^{-3}$, its intensity will only be significant for the highest $\mathrm{SO}_{2}$ column densities. 
Table 4. Selected $\mathrm{SO}_{2}$ lines in the millimeter domain.

\begin{tabular}{llcrr}
\hline \hline Line & $j_{K_{\mathrm{a}}, K_{\mathrm{c}}}$ & $\mathrm{Aij}\left(\mathrm{s}^{-1}\right)$ & $E_{\text {up }}(\mathrm{K})$ & $v(\mathrm{MHz})$ \\
\hline 1 & $3_{13}-2_{02}$ & $1.0 \times 10^{-5}$ & 7.7 & 104029.4 \\
2 & $5_{15}-4_{04}$ & $2.2 \times 10^{-5}$ & 15.7 & 135696.0 \\
3 & $5_{24}-4_{13}$ & $8.5 \times 10^{-5}$ & 23.6 & 241615.8 \\
4 & $4_{31}-4_{22}$ & $4.3 \times 10^{-6}$ & 31.3 & 160543.0 \\
\hline
\end{tabular}

The line intensity ratios between these transitions are shown in Fig. 13. We can see that the $3_{13}-2_{02} / 5_{15}-4_{04}$ line intensity ratio will be a tracer of the regions of moderate density. However, the $3_{13}-2_{02} / 5_{24}-4_{13}$ one will trace the densest regions of the cloud. No significant differences are found for these ratios as a function of the collider, ortho, or para $\mathrm{H}_{2}$. On the other hand, the line intensity ratios predicted using the He-rates are shifted towards higher densities (one order of magnitude).

To compare our results using our rates with those that could be obtained using those of Green (1995) without extrapolating the rates, we did the same calculations for a temperature of $25 \mathrm{~K}$, the lowest value provided by Green. The results indicate the same behavior as at low temperature; i.e., volume densities a factor $\simeq 10$ higher are needed for the He-rates to reproduce the predictions obtained using our rates between $\mathrm{SO}_{2}$ and o/p- $\mathrm{H}_{2}$.

\section{Summary}

We used full quantum scattering calculations to investigate rotational energy transfer in collisions of $\mathrm{SO}_{2}$ with para and ortho $\mathrm{H}_{2}$. The calculations are based on a recent, hightly accurate $5 \mathrm{D}$ potential energy surface. Rate coefficients for transitions involving the lowest 31 levels of $\mathrm{SO}_{2}$ were determined for temperatures ranging from 5 to $30 \mathrm{~K}$. It was shown that including $\mathrm{H}_{2}\left(j_{2}=2\right)$ makes a negligible contribution to para- $\mathrm{H}_{2}$ rate coefficients. Rate coefficients with para and ortho- $\mathrm{H}_{2}$ differ by a factor of two on average, and the largest are mainly the rates for collisions with ortho- $\mathrm{H}_{2}$.

The comparison between the present results and the scaled He rates of Green (1995) shows large differences that are found to exceed a factor ten for some transitions, rate coefficients with $\mathrm{H}_{2}$ being always larger than those with He. These discrepancies may be explained by the differences between the potential energy surfaces and by the approximate IOS collisional treatment used by Green for the He-rate calculations.

We used the new rates to predict the behavior of the $\mathrm{SO} 2$ lines in the centimeter and millimeter domain. For the lowest frequencies we have found that the line $1_{11}-2_{02}$ should be in absorption against the cosmic background radiation for densities below $10^{4} \mathrm{~cm}^{-3}$. The amount of absorption is directly proportional to the column density of $\mathrm{SO}_{2}$. These predictions have been confirmed with observations using the GBT telescope. We also predict that the line $4_{04}-3_{13}$ will be a maser with a maximum population inversion efficiency for densities around $5 \times 10^{3} \mathrm{~cm}^{-3}$. The observation of this line will provide strong constraints on the abundance of $\mathrm{SO}_{2}$ in the medium-density regions of cold dark clouds.

Acknowledgements. The scattering calculations were performed at the IDRISCNRS French national computing center (Institut de Développement et des Ressources en Informatique Scientifique du Centre National de la Recherche Scientifique) under project 060883 and on local work stations of the Centre Informatique of Paris Observatory. L.C.-V. was supported in 2006-2008 by the FP6 Research Training Network "Molecular Universe" under contract number MCRTN-CT-2004-512302 and in 2008-2009 by a grant of the Lavoisier Program of the French Foreign Office Ministry. J. Cernicharo thanks the Spanish MICINN for funding support under grants AYA2006-14786, AYA2009-07304, and the ASTROMOL Consolider project CSD2009-00038. M.-L. Senent also thanks the Spanish MICINN for funding support under grant AYA2005-00702. This research was also supported by the CNRS national program "Physique et Chimie du Milieu Interstellaire". We would like to thank the GBT staff for support during the observations. The authors wish to acknowledge their friend and colleague Pierre Valiron who actively participated in the work that leads to this paper. Pierre passed away on 31 August 2008, and we dedicate this paper to his memory.

\section{References}

Alexander, M. H., \& Manolopoulos, D. E. 1987, J. Chem. Phys., 86, 2044 Bachiller, R., \& Cernicharo, J. 1986, A\&A, 166, 283

Brinch, C., Crapsi, A., Hogerheijde, M. R., \& Jørgensen, J. K. 2007, A\&A, 461, 1037

Cernicharo, J., \& Bachiller, R. 1984, A\&AS, 58, 327

Cernicharo, J., \& Guélin, M., 1987, A\&A, 176, 299

Cernicharo, J., Guélin, M., \& Askne, J. 1984, A\&A, 138, 371

Cernicharo, J., Bachiller, R., \& Duvert, G. 1985, A\&A, 149, 273

Daniel, F., Cernicharo, J., Roueff, E., Gerin, M., \& Dubernet, M. L. 2007, ApJ, 667, 980

Dickens, J. E., Irvine, W. M., Snell, R. L., et al. 2000, ApJ, 542, 870

Dubernet, M.-L., Daniel, F., Grosjean, A., et al. 2006, A\&A, 460, 323

Dubernet, M.-L., Daniel, F., Grosjean, A., \& Lin, C. Y. 2009, A\&A, 497, 911

Duvert, G., Cernicharo, J., \& Baudry, A. 1986, A\&A, 164, 349

Faure, A., Crimier, N., Cecarelli, C., et al. 2007, A\&A, 472, 1029

Fuller, G. A., Ladd, E. F., \& Hodapp, K.-W. 1996, ApJ, 463, L97

Green, S. 1995, ApJS, 100, 213

Grosjean, A., Dubernet, M.-L., \& Ceccarelli, C. 2003, A\&A, 408, 1197

Hogerheijde, M. 2001, ApJ, 553, 618

Hogerheijde, M., Van Disjoeck, E., Blake, G. A., \& van Langevelde, H. J. 1997, ApJ, 489, 293

Hutson, J. M., \& Green, S. 1994, MOLSCAT computer code, version 14, Collaborative Computational Project No. 6 of the Science and Engineering Research Council, United Kingdom

Irvine, W. M., Food, J. C., \& Schloerb, F. P. 1983, A\&A, 127, L10

Jørgensen, J. K., Schöier, F. L., \& van Dishoeck, E. F. 2004, 416, 603

Lique, F., Cernicharo, J., \& Cox, P. 2006, ApJ, 653, 1342

Lique, F., Senent, M. L., Spielfiedel, A., \& Feautrier, N. 2007, J. Chem. Phys., 126,164312

Lique, F., Tobola, R., Klos, J., et al. 2008, A\&A, 567, 574

Lique, F., Spielfiedel, A., Feautrier, N., et al. 2010, J. Chem. Phys., 132, 24303

Lovas, F. J. 1978, J. Chem. Phys. Ref. Data, 7, 1445

Myers, P. C., Bachiller, R., Caselli, P., et al. 1995, ApJ, 449, L65

Ohashi, N., Hayashi, M., Ho, P. T. P., \& Momose, M. 1997, 475, 211

Palumbo, M. E., Leto, P., Siringo, C., \& Trigilio, C. 2008, ApJ, 685, 1033

Phillips, T. R., Maluendes, S., \& Green, S. 1996, ApJS, 107, 476

Pickett, H. M., Poynter, R. L., Cohen, E. A., et al. 1998, J. Quant. Spectrosc. \& Rad. Transfer, 60, 883, see also JPL Molecular Spectroscopy Catalog: http://spec.jpl.nasa.gov

Schilke, P., Benford, D. J., Hunter, T. R., Lis. D. C., \& Phillips, T. G. 2001, ApJS, 132,281

Schloerb, F. P., Irvine, W. M., Friberg, P., Hjalmarson, A., \& Hoglund, B. 1983, ApJ, 264, 161

Snyder, L. E., Hollis, J. M., Ulich, B. L., et al. 1975, ApJ, 198, L81

Spielfiedel, A., Senent, M.-L., Dayou, F., et al. 2009, J. Chem. Phys., 131, 014305

Takakuwa, S., Mikami, H., Saito, M., \& Hirano, N. 2000, ApJ, 542, 367

Troscompt, N., Faure, A., Wiesenfeld, L., Ceccarelli, C., \& Valiron, P. 2009, A\&A, 493, 687

Valiron, P., \& McBane, G. C. 2008, MPI and OpenMP extension of MOLSCAT; program repository on:

http://wwW-laog.obs.ujf-grenoble.fr/ valiron/molscat/

Valiron, P., Wernli, M., Faure, A., et al. 2008, J. Chem. Phys., 129, 134306

Wernli, M., Wiesenfeld, L., Faure, A., \& Valiron, P. 2007a, A\&A, 464, 1147; A\&A, 475, 391

Wernli, M., Wiesenfeld, L., Faure, A., \& Valiron, P. 2007b, A\&A, 475, 391

Woon, D. E., \& Dunning Jr., T. H. 1994, J. Chem. Phys., 100, 2975

William, H. L., Mas, E. M., Szalewicz, K., \& Jeziorski, B. 1995, J. Chem. Phys., 103, 7374 